\title{
Performance analysis of CSMA in an unslotted cognitive radio network with licensed channels and unlicensed channels
}

\author{
Dong Bi Zhu' and Bong Dae $\mathrm{Choi}^{2^{*}}$
}

\begin{abstract}
We consider a carrier sense multiple access(CSMA) in an unslotted cognitive radio network under a mixed spectrum environment of licensed channels and unlicensed channels. In this system, primary users use only licensed channels, while secondary users use unlicensed channels and opportunistically can use licensed channels unused by primary users temporally. If an arriving secondary user(SU) does not find any idle channels, then the SU either enters the retrial group with probability $q$ for later retrial or gives up its service and leaves the system with probability $1-q$. SUs in the retrial group retry independently after exponentially distributed random time. We analyze the system by continuous time Markov chain(CTMC) with level dependent QBD structure and obtain the steady state probability of the system by matrix analytic method. In numerical results, we compare the performance of two systems with retrial $(q>0)$ and without retrial $(q=0)$. It is shown that the retrial phenomenon of SUs has an impact on the performance of SUs in cognitive radio networks.
\end{abstract}

Keywords: Cognitive Radio Network, CSMA; Retry SU, Matrix Analytic Method, Throughput, Loss probability

\section{Introduction}

Advances in wireless communication systems and development of new services have significantly increased the demand for more frequency bands. Recently, the FCC has reported that the licensed bands are vastly underutilized [1]. To overcome spectrum scarcity, licensed spectrum bands need to be more intelligently utilized. For this purpose, cognitive radios have recently emerged as a promising technique to improve the utilization of the existing radio spectrum. Cognitive radio improves the spectrum efficiencies by enabling SUs to opportunistically access the channels unused by PUs.

There have been many studies on the opportunistic spectrum access for a cognitive radio network. Cognitive radio networks can be classified as single channel(e.g., $[2,4,5,7])$ and multi-channel(e.g., $[3,6,8])$. Both networks further can be classified a slotted structure(e.g., $[2,3,6])$ and an unslotted structure(e.g., [7-9]) where the slotted structure means the time axis for network is divided by

\footnotetext{
* Correspondence: queue@korea.ac.kr

${ }^{2}$ Department of Mathematics and Telecommunication Mathematics Research Center, Korea University, Seoul, Korea

Full list of author information is available at the end of the article
}

time slots and all PUs and SUs are synchronized at the time slot.

Most of these studies deal with temporal use of the SUs on licensed bands. Multiple accesses of SUs on a mixed environment of licensed and unlicensed bands have been investigated [10-12]. H. Al-Mahdi et. al. [12] investigated CSMA in an unslotted cognitive radio networks under a mixed spectrum environment of licensed and unlicensed bands where the blocked SUs and the preempted SUs are forced to leave the system forever when there are no idle channels in the system. But in practical situation, the blocked SUs and the preempted SUs may do not leave the system forever and try to continue their services after random amount of time. This is a motivation of our work.

In this paper, we investigate the same model in [12] with an additional retrial phenomenon: the blocked SUs and the preempted SUs either enter a retrial group with probability $q$ or leave the system with probability $1-q$ if all channels are busy. We investigate the effect of the retrial phenomenon of SUs on the system performance of an unslotted cognitive radio network. We analyze the system by CTMC with a level dependent QBD structure 
and obtain the steady state probability of the system using matrix analytic method. Note that, when $q=0$, our model reduces to the system model in [12].

The rest of this paper is organized as follows. In Section 2, the operation of CSMA in unslotted cognitive radio networks with a mixed spectrum environment is described in details. In Section 3, we analyze the performance of the system by using matrix analytic method. Numerical examples are presented in section 4 and conclusions are given in section 5 .

\section{System model}

We consider CSMA in the unslotted cognitive radio networks with a mixed spectrum environment of $c_{1}$ primary channels and $c_{2}$ secondary channels(See Figure 1). PUs can use only $c_{1}$ primary channels but SUs can use $c_{2}$ secondary channels and can opportunistically use primary channels unused by PUs. PUs have preemptive priorities over SUs on primary channels. We assume that PUs and SUs arrive according to Poisson processes with rate $\lambda_{1}$ and $\lambda_{2}$ independently, the transmission times of messages for PUs and SUs are exponentially distributed with mean $\mu_{1}{ }^{-1}$ and $\mu_{2}{ }^{-1}$, respectively.

On the arrival of a PU, if there are idle primary channels, the PU occupies one of those idle channels and send its message. If there is no idle primary channel but at least one primary channels are occupied by SUs, the arriving PU selects and preempts one of the primary channels occupied by SUs. If all primary channels are busy with PUs, the arriving PU is queued in the finite buffer with the size $N-c_{1}\left(N \geq c_{1}\right)$.

A SU always senses the channels before each message transmission. It is assumed that the sensing period of the channel is negligible. An arriving SU first senses unlicensed channels. If there are idle secondary channels, then the SU occupies one of the idle secondary channels and transmits a message and leaves the system after its transmission. When the arriving SU does not find idle secondary channels, the SU senses the primary channels and transmits its message on an idle primary channel if any, otherwise the SU either enters the retrial group with probability $q$ for later retrial or give up its service and leaves the system with probability $1-q$.

When a SU is preempted by a PU, the SU performs spectrum handoff to a secondary channel. Spectrum handoff procedures aim to help SUs find another idle channel to send its message. If the preempted SU finds idle secondary channels, the SU handoffs to one of those idle channels, otherwise the SU either enters the retrial group with probability $q$ or leaves the system with probability $1-q$.

SUs in the retrial group retry to the system in order to transmit their messages. The retrial time of SUs is defined as the amount of time between two consecutive retrials made by a $\mathrm{SU}$ and is assumed to be independent of all previous retrial times. We assume that the retrial times are exponentially distributed with mean $v^{-1}$. When a SU in the retrial group retries to the system, the SU repeats the same procedure as an arriving $\mathrm{SU}$ does.

\section{Performance analysis of the system}

In this section, we analyze CSMA in an unslotted cognitive radio network with a mixed spectrum environment of licensed and unlicensed channels by using matrix analytic method.

Let

$N_{r}(t)=$ the member of SUs in the retrial group at time $t$,

$N_{1}(t)=$ the total number of PUs in service and PUs in the buffer at time $t$,

$N_{2}(t)=$ the number of SUs in service at time $t$.

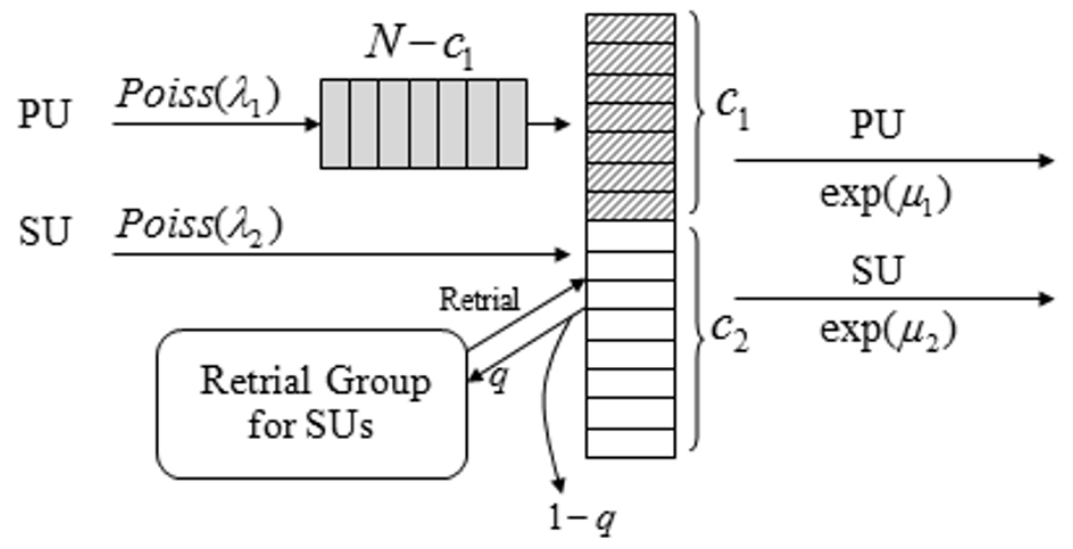

Figure 1 The system model 
Then $\left\{\left(N_{r}(t), N_{1}(t), N_{2}(t)\right) \mid t \geq 0\right\}$ forms 3-dimensional Markov process with state space

$$
\mathcal{S}=\left\{(k, n, j) \mid 0 \leq k, 0 \leq n \leq N, 0 \leq j \leq \max \left\{c-n, c_{2}\right\}\right\}
$$

where $c=c_{1}+c_{2}$.

Let the elements of $\mathcal{S}$ be ordered lexicographically. The infinitesimal generator $\mathbf{Q}$ of the Markov process has a level dependent infinite QBD structure as follows.

$$
\mathbf{Q}=\left[\begin{array}{cccccccc}
\mathbf{B}_{1} & \mathbf{A} & 0 & \cdots & 0 & 0 & 0 & \cdots \\
\mathbf{B}_{2} \mathbf{B}_{1}-\boldsymbol{\Gamma} & \mathbf{A} & \cdots & 0 & 0 & 0 & \cdots \\
0 & 2 \mathbf{B}_{2} & \mathbf{B}_{1}-2 \boldsymbol{\Gamma} & \cdots & 0 & 0 & 0 & \cdots \\
\vdots & \vdots & \vdots & \vdots & \vdots & \vdots & \vdots & \vdots \\
0 & 0 & 0 & \cdots & k \mathbf{B}_{2} & \mathbf{B}_{1}-k \Gamma & \mathbf{A} & \cdots \\
\vdots & \vdots & \vdots & \vdots & \vdots & \vdots & \vdots & \vdots
\end{array}\right]
$$

The entries of $\mathbf{Q}$ are given by following block matrices.

i) $\mathbf{A}$ is the transition rate matrix from level $k$ to level $k+1$, given by

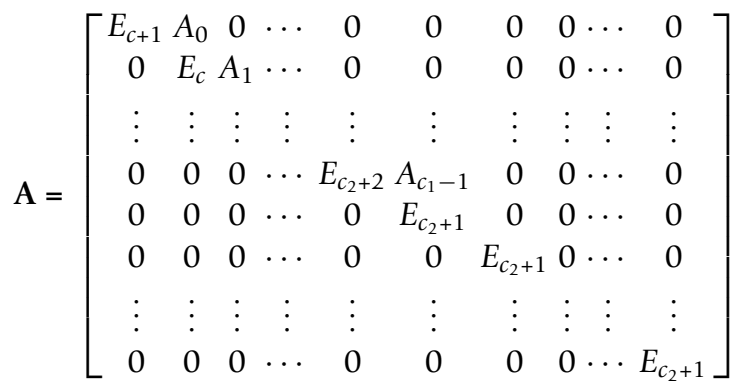

where $E_{n}=\operatorname{Diag}\left(0, \ldots, 0, \lambda_{2} q\right)$ is an $n \times n$ diagonal matrix and $A_{n}, 0 \leq n \leq c_{1}-1$ are $(c-n+1) \times(c-n)$ matrices as follows.

$$
A_{n}=\left[\begin{array}{cccc}
0 & 0 & \cdots & 0 \\
0 & 0 & \cdots & 0 \\
\vdots & \vdots & \ddots & \vdots \\
0 & 0 & \cdots & 0 \\
0 & 0 & \cdots & \lambda_{1 q}
\end{array}\right]
$$

ii) $k \mathbf{B}_{2}$ is the transition rate matrices from level $k$ to level $k-1 . \mathbf{B}_{2}$ is given by

$$
\mathbf{B}_{2}=\operatorname{Diag}\left(B_{0}^{(2)}, B_{1}^{(2)}, \ldots, B_{N}^{(2)}\right)
$$

where $B_{n}^{(2)}, 0 \leq n \leq c_{1}$ are $(c-n+1) \times(c-n+1)$ matrices given by

$$
B_{n}^{(2)}=\left[\begin{array}{ccccc}
0 & v & 0 & \cdots & 0 \\
0 & 0 & v & \cdots & 0 \\
\vdots & \vdots & \vdots & \vdots & \vdots \\
0 & 0 & 0 & \cdots & v \\
0 & 0 & 0 & \cdots & (1-q) v
\end{array}\right]
$$

and $B_{c_{1}}^{(2)}=B_{c_{1}+1}^{(2)}=\cdots=B_{N}^{(2)}$.

iii) $\mathrm{B}_{1}-k \Gamma$ is the transition rate matrices in level $k$. The matrix $\Gamma$ is given by

$$
\boldsymbol{\Gamma}=\operatorname{Diag}\left(\Gamma_{0}, \Gamma_{1}, \cdots, \Gamma_{N}\right)
$$

where $\Gamma_{n}=\operatorname{Diag}(v, v, \ldots, v,(1-q) v)\left(0 \leq n \leq c_{1}\right)$ is $(c-n$ $+1) \times(c-n+1)$ diagonal matrix and $\Gamma_{c_{1}}=\Gamma_{c_{1}+1}=\cdots=\Gamma_{N} . \mathrm{B}_{1}$ has following structure:

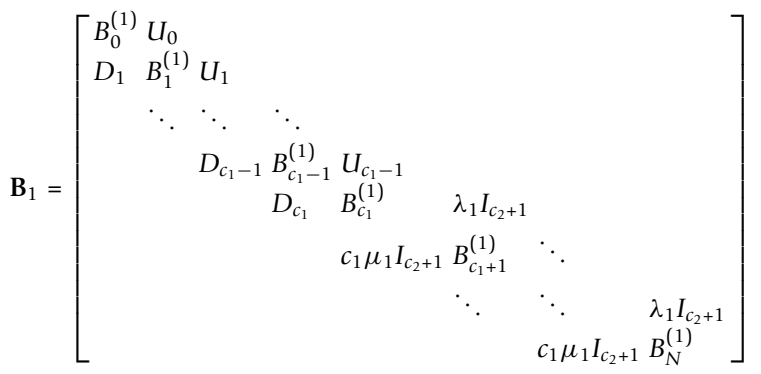

where $I_{n}$ is an $n \times n$ identity matrix, $U_{n}, 0 \leq n \leq c_{1}-1$ are $(c-n+1) \times(c-n)$ matrices and $D_{n}, 1 \leq n \leq c_{1}$ are $(c-n+1) \times(c-n+2)$ matrices as follows:

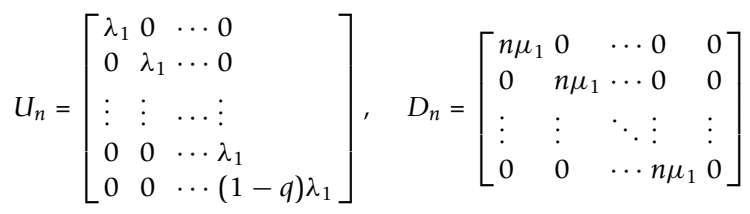

Let $d_{n} \triangleq \max \left\{c-n, c_{2}\right\} \cdot B_{n}^{(1)}, 0 \leq n \leq N$ are $\left(d_{n}+1\right) \times$ $\left(d_{n}+1\right)$ square matrices, has the structure

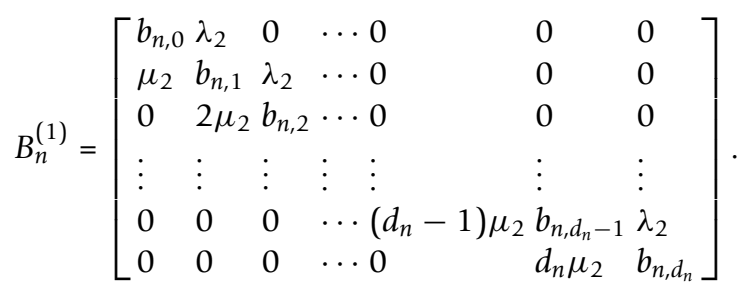

where the diagonal elements $b_{n, j}$ are given by

$$
b_{n, j}=\left\{\begin{array}{cl}
-\left[\lambda_{1}+\lambda_{2}+\min \left\{n, c_{1}\right\} \mu_{1}+j \mu_{2}\right], & \text { if } 0 \leq n \leq N-1,0 \leq j<d_{n} \\
-\left[\lambda_{1}+q \lambda_{2}+\min \left\{n, c_{1}\right\} \mu_{1}+j \mu_{2}\right], & \text { if } 0 \leq n \leq N-1, j=d_{n \prime} \\
-\left[\lambda_{2}+c_{1} \mu_{1}+j \mu_{2}\right], & \text { if } n=N, 0 \leq j<c_{2} \\
-\left[q \lambda_{2}+c_{1} \mu_{1}+j \mu_{2}\right], & \text { if } n=N, j=c_{2} .
\end{array}\right.
$$

Note that the generator $\mathbf{Q}$ is a level dependent. It is obvious that the system is stable if $q$ is less than 1 . Let $\Pi$ be the steady state probability vector, which is the 
unique solution of linear equations $\Pi \mathbf{Q}=0, \Pi \mathbf{e}_{\infty}=1$; where $\mathbf{e}_{\infty}$ is an infinite dimensional column vector whose elements are all equal to 1 . We partition $\Pi$ as $\boldsymbol{\Pi}=\left(\Pi_{0}, \Pi_{1}, \Pi_{2}, \ldots\right)$ where $\Pi_{k}=\left(\Pi_{k, 0}, \Pi_{k, 1}, \ldots, \Pi_{k, N}\right)$, $\Pi_{k, n}=\left(\pi_{k, n, 0}, \pi_{k, n, 1}, \cdots, \pi_{k, n, d_{n}}\right)$ and $\pi_{k, n, j}$ is the probability that Markov chain is in state $(k, n, j)$ in the steady state. We can obtain the steady state probability vector $\Pi$ by matrix analytic method(See an Appendix for detailed derivation).

The main performance measures of cognitive radio network are loss probability $P_{l}$ and the throughput $T_{s}$ of SUs.

Let $\pi_{. n, j} \triangleq \sum_{k=0}^{\infty} \pi_{k, n, j}$ is the probability that there are $n$ PUs in the system and $j$ SUs in service. Note that, since $n+d_{n} \geq c, \sum_{n=0}^{N} \pi_{. n, d_{n}}$ is the probability that all $c$ channels are busy.

The loss probability $P_{l}$ of SUs is defined by the ratio of the loss rate of SUs to the arrival rate of SUs. Loss of SUs occurs by the following three kinds of events:

- a new SU is blocked and then gives up its service and thus its loss rate is given by

$\lambda_{2}(1-q) \sum_{n=0}^{N} \pi_{\cdot n, d_{n}}$

where $\sum_{n=0}^{N} \pi_{\cdot n, d_{n}}$ is the probability that all $c$ channels are busy.

- a retrial SU is blocked and then gives up its service and thus its loss rate is given by

$\sum_{k=1}^{\infty} \sum_{n=0}^{N} k \mathcal{V}(1-q) \pi_{k, n, d_{n}}$

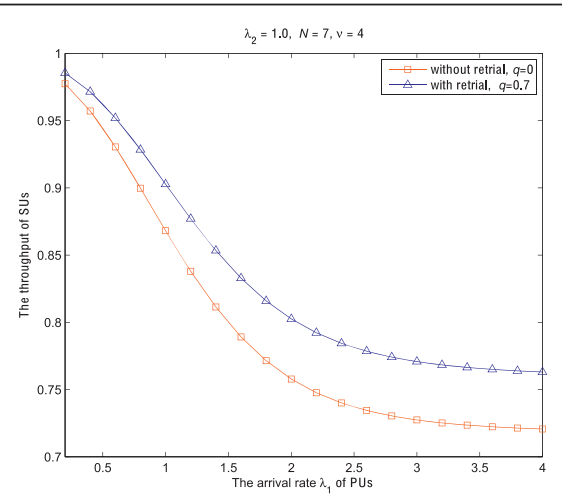

(a) The throughput of SUs where $k v$ is a retrial rate when there are $k$ SUs in a retrial group.

- a SU is preempted by a PU and then gives up its service and thus its loss rate is given by

$\lambda_{1}(1-q) \sum_{n=0}^{c_{1}-1} \pi_{\cdot n, d_{n}}$

where a SU is preempted when all $c$ channels are busy and at least one SU occupy primary channel.

Hence the total loss rate of SUs is

$$
(1-q)\left(\lambda_{2} \sum_{n=0}^{N} \pi_{. n, d_{n}}+\sum_{k=1}^{\infty} \sum_{n=0}^{N} k \mathcal{V} \pi_{k, n, d_{n}}+\lambda_{1} \sum_{n=0}^{c_{1}-1} \pi_{. n . d_{n}}\right) .
$$

Therefore the loss probability of SUs is given by

$$
P_{l}=\frac{1-q}{\lambda_{2}}\left(\lambda_{2} \sum_{n=0}^{N} \pi_{n, d_{n}}+\sum_{k=1}^{\infty} \sum_{n=0}^{N} k \mathcal{V} \pi_{k, n, d_{n}}+\lambda_{1} \sum_{n=0}^{c_{1}-1} \pi_{n, d_{n}}\right) .
$$

The throughput $T_{s}$ of SUs is defined as the number of SUs who successfully transmit their messages per unit time, i.e., we have

$$
T_{s}=\lambda_{2}\left(1-P_{l}\right) .
$$

\section{Numerical results}

In this section, we present numerical examples to investigate the performance evaluation of CSMA in unslotted cognitive radio networks with a mixed spectrum environment. We set the parameters $c_{1}=4, c_{2}=3$ channels, $\mu_{1}^{-1}=2, \mu_{2}^{-1}=2.5(\mathrm{sec})$.

In Figure 2 and Figure 3, we compare the throughput and loss probability of SUs between the case with retrials $(q=0.7)$ and the case without retrials $(q=0)$.

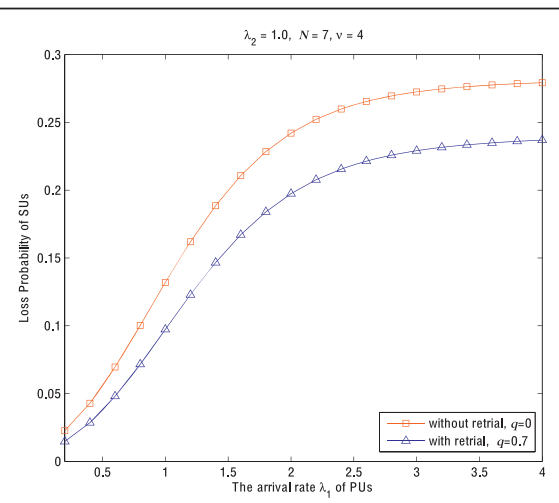

(b) The loss probability of SUs

Figure 2 Performance of SUs versus the arrival rate $\lambda_{1}$ of Pus. 
Figure 2(a) depicts the throughput of SUs versus the arrival rate $\lambda_{1}$ of PUs. As expected, the throughput of SUs decreases as the arrival rate $\lambda_{1}$ of PUs increases. We see that the throughput of SUs in the case with retrials is larger than that in the case without retrials, because the retrial phenomenon of SUs leads to higher channel utilization of SUs. Figure 2(b) shows the loss probability of SUs increases as the arrival rate $\lambda_{1}$ of PUs increases. We also see that the loss probability of SUs in the case without retrials is larger than that in the case with retrials because all blocked SUs are lost in the model without retrials. Figure $3(\mathrm{a})$ and $3(\mathrm{~b})$ depict the throughput of SUs and loss probability of SUs versus the arrival rate $\lambda_{2}$ of SUs. Figure 2 and Figure 3 show the retrial phenomenon of SUs has an impact on the performance of CSMA scheme in unslotted cognitive radio networks.

Figure 4(a) and 4(b) depict the throughput of SUs and the loss probability of SUs versus the buffer size $N-c_{1}$ of PUs. It is show that the throughput of SUs decreases and the loss probability of SUs increases as the buffer size $N-c_{1}$ of PUs increases. But when the buffer size larger than certain point, the effect of PU's buffer size on the performance of SUs is very small because when PUs wait in the buffer, SUs can't use primary channels and only can use secondary channels.

Figure 5(a) and 5(b) depict the throughput of SUs and the loss probability of SUs versus the retrial rate $v$ of PUs. It is show that the throughput of SUs decreases and the loss probability of SUs increases as the retrial rate $v$ of PUs increases because the loss rate of SUs increases.

\section{Conclusions}

We have considered CSMA in an unslotted cognitive radio network under a mixed spectrum environment of licensed and unlicensed channels and investigated the effect of retrial phenomenon of SUs. We analyze the system by CTMC with a level dependent QBD structure and obtain the steady state probability of the system using matrix analytic method, and then obtain

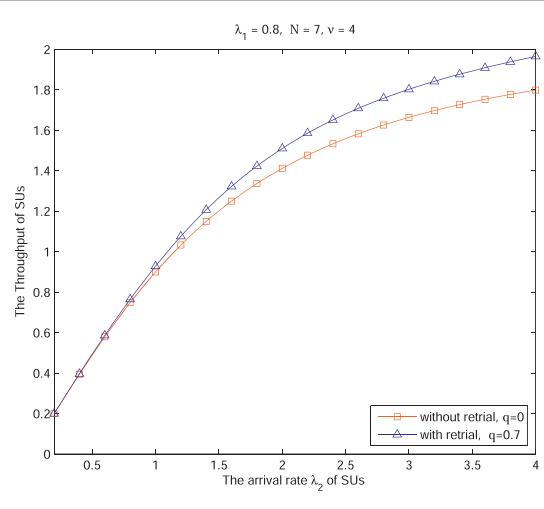

(a) The throughput of SUs

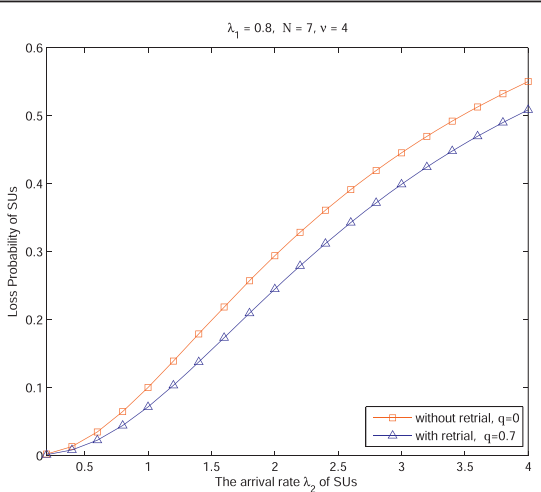

(b) The loss probability of SUs

Figure 3 Performance of SUs versus the arrival rate $\lambda_{2}$ of Pus.

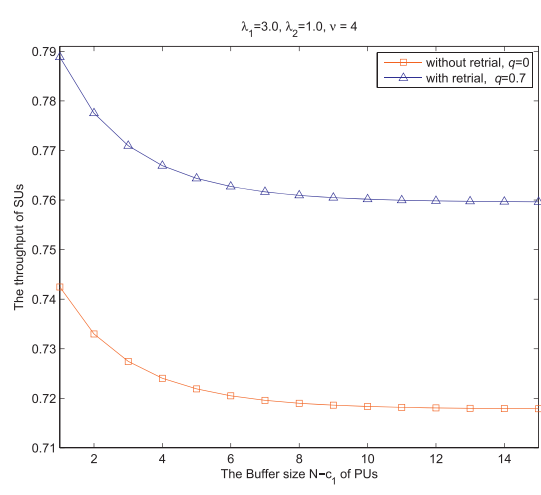

(a) The throughput of SUs

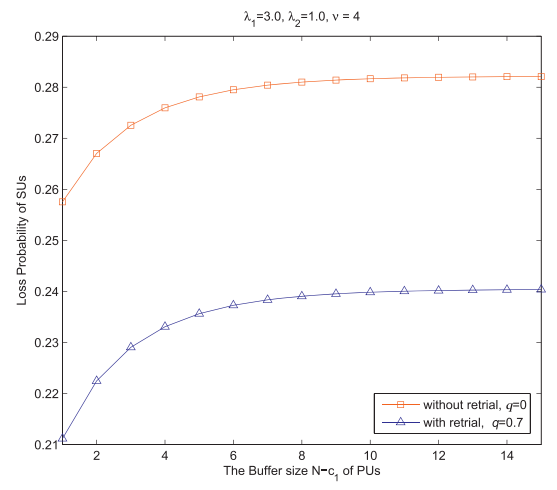

(b) The loss probability of SUs

Figure 4 Performance of SUs versus the buffer size $\mathrm{N}-\mathrm{C}_{1}$ of Pus. 


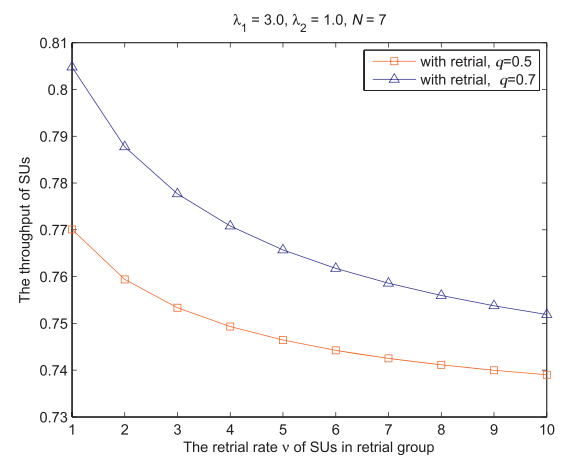

(a) The throughput of SUs

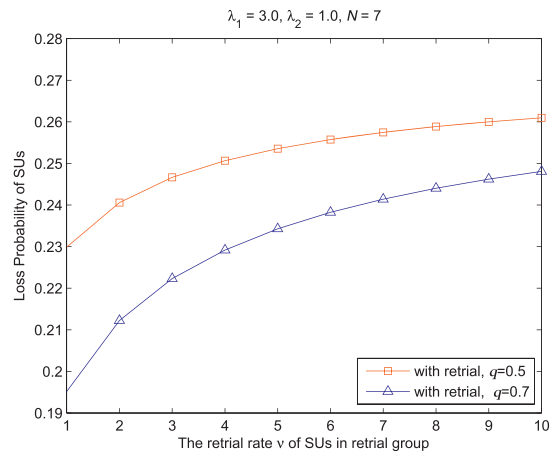

(b) The loss probability of SUs

Figure 5 Performance of SUs versus retrial rate $v$ of SUs in retrial group.

performance measures of SU such as the loss probability of SUs and the throughput of SUs. Numerical results show that the retrial phenomenon of SUs have an impact on the loss probability and throughput of SUs in cognitive radio networks under a mixed spectrum environments of licensed and unlicensed channels.

\section{Appendix}

The generator $\mathbf{Q}$ is the level dependent quasi birth-anddeath matrix. Let $\boldsymbol{\Pi}$ be the steady state probability vector, which is the unique solution of linear equations IQ $=0, \boldsymbol{\Pi} \mathbf{e}_{\infty}=1$ where $\mathbf{e}_{\infty}$ is an infinite dimensional column vector whose elements are all equal to 1 . To obtain the steady state probabilities, we use Neuts' approximation method (Neuts and Rao [13]). We choose appropriate $K$ by this method and assume that only $K$ calls among the retrial calls in the retrial group can retry for the service even if there are retrial calls greater than $K$ in the current retrial group.

Then the infinitesimal generator $\tilde{\mathbf{Q}}$ of the approximated system is given by

$$
\tilde{\mathbf{Q}}=\left[\begin{array}{lllllll}
\mathbf{B}_{1} \mathbf{A}_{0} & & & & & \\
\mathbf{B}_{2} \mathbf{B}_{1}-\Gamma & \mathbf{A}_{0} & & & & \\
2 \mathbf{B}_{2} & \mathbf{B}_{1}-2 \Gamma \mathbf{A}_{0} & & & \\
& \ddots & \ddots & \ddots & & \\
& & K \mathbf{B}_{2} & \mathbf{B}_{1}-K \Gamma \mathbf{A}_{0} & \\
& & & \mathbf{A}_{2} & \mathbf{A}_{1} \mathbf{A}_{0} & \\
& & & & \mathbf{A}_{2} \mathbf{A}_{1} \mathbf{A}_{0} \\
& & & & & \ddots & \ddots
\end{array}\right]
$$

where $A_{2} \equiv K_{B_{2}}, A_{1} \equiv \mathbf{B}_{1}-\mathbf{K} \boldsymbol{\Gamma}$.

We can easily obtain the following results by Matrixgeometric method(see Neuts [14]). The unique steadystate probability vector $\tilde{\boldsymbol{\Pi}}$ satisfying $\tilde{\boldsymbol{\Pi}} \tilde{\mathbf{Q}}=0, \tilde{\boldsymbol{\Pi}} e_{\infty}=1$ are given by

$$
\tilde{\Pi}_{k}=\left\{\begin{array}{cl}
\tilde{\Pi}_{K} H_{K} H_{K-1} \cdots H_{k+1}, & \text { for } 0 \leq j<K \\
\tilde{\Pi}_{K} R^{k-K}, & \text { for } k \geq K
\end{array}\right.
$$

The vector $\tilde{\Pi}_{K}$ is obtained by solving

$$
\left\{\begin{array}{c}
\tilde{\Pi}_{K}\left[H_{K} \mathbf{A}_{0}+\mathbf{A}_{1}+R \mathbf{A}_{2}\right]=0 \\
\tilde{\Pi}_{K}\left[\sum_{k=0}^{K-1}\left(H_{K} H_{K-1} \cdots H_{K+1}\right)+(I-R)^{-1}\right] \mathrm{e}=1
\end{array}\right.
$$

where

$$
\begin{aligned}
& H_{0}=0, \\
& H_{m}=-\left(m B_{2}\right)\left[H_{m-1} A_{0}+B_{1}-(m-1) \Gamma\right]^{-1}, \text { for } 1 \leq n \leq K
\end{aligned}
$$

and $R$ is the minimal nonnegative matrix solution to the matrix-quadratic equation

$$
R^{2} \mathbf{A}_{2}+R \mathbf{A}_{1}+\mathbf{A}_{0}=0 .
$$

Note that the matrix $R$ is approximated by the following iteration

$$
\begin{aligned}
R(0) & =0 \\
R(n+1) & =-\mathbf{A}_{0} \cdot \mathbf{A}_{1}^{-1}-R^{2}(n) \cdot \mathbf{A}_{2} \mathbf{A}_{1}^{-1}, \text { for } n \geq 0
\end{aligned}
$$

Iterations will be continued until $\max _{i, j} \mid[R(n+1)]_{i j}$ $[R(n)]_{i j} \mid<\varepsilon$ is satisfied. $[R(n)]_{i j}$ is the $i \times j$ th elements of $n$th iteration $R(n)$.

\section{Acknowledgements}

This research is supported by the Korea Foundation for Advanced Studies' International Scholar Exchange Fellowship for the academic year of 20092010, and the MKE(Ministry of Knowledge Economy), Korea, under the ITRC (Information Technology Research Center) support program supervised by the NIPA(National IT Industry Promotion Agency), Korea.

\section{Author details}

${ }^{1}$ Department of Electronics and Communication Engineering, College of Engineering, YanBian University, YanJi, China ${ }^{2}$ Department of Mathematics and Telecommunication Mathematics Research Center, Korea University, Seoul, Korea 


\section{Conflicts of interests}

The authors declare that they have no competing interests.

Received: 22 January 2011 Accepted: 13 January 2012

Published: 13 January 2012

\section{References}

1. FCC, "Spectrum Policy Task Force Report". No. 02-155 (Nov 2002)

2. Ping Wang, Niyato Dusit, Jiang Hai, "Voice Service Support over Cognitive Radio Networks". Communications, 2009. ICC '09. IEEE International Conference on 1-5 (June 2009)

3. Su Hang, Zhang Xi, "Cross-Layer Based Opportunistic MAC Protocols for QoS Provisionings Over Cognitive Radio Wireless Networks". IEEE Journal on Selected Areas in Communications. 26(1), 118-129 (Jan 2008)

4. Han Bae Yun, S Alfa Attahiru, Dae Choi Bong, " Analysis of a contentionbased opportunistic spectrum access under general channel activity model". Performance Evaluation. 68(3), 271-289 (March 2011). doi:10.1016/j. peva.2010.12.003

5. Han Bae Yun, S Alfa Attahiru, Dae Choi Bong, "Performance Analysis of Modified IEEE 802.11-Based Cognitive Radio Networks". IEEE Communications Letters. 14(10), 975-977 (Oct 2010)

6. JW Chong, Y Sung, DK Sung, "RawPEACH:Multiband CSMA/CA-Based Cognitive Radio Networks". Journal of Communications and Networks. 11(2), 174-185 (April 2009)

7. Senhua Huang, Liu Xin, Ding Zhi, "Opportunistic Spectrum Access in Cognitive Radio Networks". INFOCOM 2008. The 27th Conference on Computer Communications. IEEE 1427-1435 (April 2008)

8. WM Wong Eric, Heng Foh Chuan, "Analysis of cognitive radio spectrum access with finite user population". IEEE Communications Letters. 13(5), 294-296 (May 2009)

9. DB Zhu, JS Park, BD Choi, "Performance Analysis of an unslotted CSMA in the Multi-Channel Cognitive Radio Networks". 5th International Conferences on Queueing Theory and its Applications QTNA2010 (July 2010)

10. Y Xing, R Chandramouli, S Mangold, S Shankar N, "Dynamic spectrum access in open spectrum wireless networks". IEEE Journal on Selected Areas in Communications. 24(3), 626-637 (Mar 2006)

11. L Wang, A Chen, DS Wei, A cognitive MAC protocol for QoS provisioning in overlaying ad hoc networks. 4th IEEE Consumer Communications and Networking Conference (CCNC 2007) 1139-1143 (Jan 2007)

12. Al-Mahdi Hassan, Kalil Mohamed A, Liers Florian, Mitschele-Thiel Andreas, "Increasing Spectrum Capacity for Ad Hoc Networks using Cognitive Radios: An Analytical Model". IEEE Communications Letters. 13(9), 676-678 (Sep 2009)

13. MF Neuts, BM Rao, "Numerical investigation of a multiserver retrial model". Queueing Systems. 7, 169-190 (1990). doi:10.1007/BF01158473

14. MF Neuts, "Matrix-Geometric Solutions in Stochastic Models", (The John Hopkins University Press, Baltimore, 1981)

doi:10.1186/1687-1499-2012-12

Cite this article as: Zhu and Choi: Performance analysis of CSMA in an unslotted cognitive radio network with licensed channels and unlicensed channels. EURASIP Journal on Wireless Communications and Networking 2012 2012:12.

\section{Submit your manuscript to a SpringerOpen ${ }^{\mathcal{O}}$ journal and benefit from:}

- Convenient online submission

- Rigorous peer review

- Immediate publication on acceptance

- Open access: articles freely available online

- High visibility within the field

- Retaining the copyright to your article

Submit your next manuscript at $\gg$ springeropen.com 